\title{
Influence des contraintes sur la perméabilité du granite : étude en laboratoire et méthode d'interprétation des essais
}

F. SKOCZYLAS

École Centrale de Lille

URA CNRS 1441

Cité Scientifique BP 48

59651 Villeneuve-d'Ascq

Cedex

\section{J.-P. HENRY}

Laboratoire

de Mécanique de Lille

USTL

URA CNRS 1441

59655 Villeneuve-d'Ascq

Cedex

\section{J.-C. GROS}

Institut de Protection et de Sûreté Nucléaire

DPEI/SERGD, BP 6 92265 Fontenay-aux-Roses Cedex

Les résultats obtenus à partir de nombreux essais de perméabilité en forage dans les massifs rocheux fracturés, on montré que la fracturation ouverte a un rôle déterminant sur les écoulements et, par voie de conséquence, que les caractéristiques hydrauliques du milieu décroissent quand la profondeur augmente.

S'il est admis que la fracturation augmente près de la surface (fractures de retrait au moment du refroidissement du granite, fractures de décompression...), les études structurales réalisées dans les forages profonds et dans les galeries de reconnaissance ont montré que la densité de fracturation n'est pas influencée de façon significative par la profondeur. L'ouverture des fractures hydrauliquement conductrices dépend étroitement des contraintes effectives qui leur sont appliquées dans le massif. Une Ioi exponentielle empirique entre la perméabilité des fractures et la contrainte normale effective qu'elles supportent, est utilisée généralement pour traduire leur comportement hydraulique en fonction de la profondeur (C. Louis).

Nous nous proposons dans cet article d'étudier le comportement hydraulique de blocs de granite, respectivement sain et parcouru par une fissure, dans un appareil triaxial vrai de grande capacité, pour vérifier cette loi empirique et pour mettre au point une méthode d'interprétation qui prenne en compte la répartition non uniforme des contraintes autour du forage dans lequel sont effectuées les mesures de perméabilité. Cette méthode, qui donne une bonne corrélation entre les mesures en régime permanent et en régime transitoire de type " pulse-tests $n$, sera étendue par la suite à l'interprétation d'essais de perméabilité en forage, dans les massifs fracturés.

Les essais ont montré l'influence de la fissuration sur la perméabilité, même pour des valeurs élevées de la contrainte verticale.

Au cours du chargement, l'approximation de la perméabilitẻ par une loi exponentielle est satisfaisante, alors que le déchargement se traduit par une hystérésis.

Pour des rapports de contraintes, sh/sv élevés, la perméabilité est relativement indépendante de la contrainte verticale.

\section{Influence of stresses on granite permeability : laboratory research and trial interpretation method}

The results obtained from numenous borebole permeability trials in fractured rocky massifs demonstrated that open fracturation played a key role in flowing and, consequently, that the milieu's hydravilic characteristics decreased with increasing depth. Although it is commonly accepted that fracturation increases closer the surface (shrinkage fractures on granite cooling. clecompression fractures.... structural surveys carried out in deep drilling and exploration galleries have shown that depth does not significantly affect fracturation density. The opening of hydrologically conductive fractures is closely dependant on the effective stresses applied on the massif. And empirical exponential law relating fractures permeability to normal effective stress tolerated is generally used to express the hydratlic behaviour of fractures according to depth (C, Louis).

In the present paper, we propose to examine the hydraulic behaviour of sound and cracked granite blocks using a high capacity true triaxial loading system to ascertain this empirical law and develop an interpretative method which takes into account the non-uniform distribution of stresses around the borehole in which the permeability trials are carried out. The method, which gave good correlation between steady state measurements and unsteady-state measurements of the pulse-test type, will later be extended to interpreting borehole permeability trials in fractured massifs. The trials showed the influence of cracking on permeability, even for very high value of vertical

stresses.

When stresses were applied, an approximation of permeability using an exponential law was satisfactory, whereas relieving it resulted in hysteresis. In high sh/sy stresses ratios, permeability was relatively independant of the vertical stress. 


\section{NOTATIONS}

$d_{p}$

$\mathrm{G}_{\mathrm{K}}$

gradient de pression au bord du forage.

$\mathrm{G}_{\mathrm{h}} \quad$ : module de cisaillement drainé.

$\mathrm{K}_{\mathrm{p}} \quad$ : module d'incompressibilité drainé,

$\mathrm{K}: \quad$ : module d'incompressibilité de la matrice rocheuse.

$\mathrm{K}_{\mathrm{n}} \quad$ : module d'incompressibilité de l'eau.

$\mathrm{K} \quad$ : compressibilitè du montage.

k : conductivité hydraulique de la roche.

K : perméabilité intrinsèque de la roche.

K : perméabilité de la roche non contrainte.

$\mathrm{K}_{\mathrm{i}} \quad$ : perméabilité au bord du forage.

$\mathrm{k}_{\mathrm{o}} \quad$ : coefficient de poussée.

$\mathrm{L}_{\mathrm{I}} \quad$ : Longueur du forage,

m : masse fluide.

M : module de Biot.

P : pression interstitielle.

$\mathrm{P}_{c} \quad$ : pression de confinement.

$\mathrm{P}_{\mathrm{P}} \quad$ : pression d'injection.

$\mathrm{P}(\mathrm{t}) \quad$ : pression d'injection pendant un pulse-test.

$\mathrm{P}_{\mathrm{o}} \quad$ : pression au rayon $\mathrm{R}_{\mathrm{6}}$.

Q : débit d'injection.

$\mathrm{R}_{\mathrm{H}} \quad$ : rayon du forage.

$\mathrm{R}_{\mathrm{o}} \quad$ : rayon de drainage.

U(r) : champ de déplacement radial.

$\mathrm{V} \quad$ : vitesse de diffusion.

$\alpha \quad$ : paramètre expérimental.

$\beta \quad$ : coefficient de Biot.

$\Phi \quad$ : porosité de la roche.

$\lambda \quad$ : constante d'intégration.

$\lambda_{\mathrm{n}} \quad$ : coefficient de Lamé drainé,

$\mu \quad$ : viscosité du fluide.

$v_{b} \quad:$ coefficient de Poisson drainé.

$\Theta \quad$ : volume de la chambre d'injection.

$p \quad \quad$ : masse spécifique du fluide à $\mathrm{P}$.

$\rho_{0} \quad:$ masse spécifique à $\mathrm{P}_{\mathrm{n}}$.

$\sigma_{\mathrm{h},} \sigma_{\mathrm{h}^{1}}, \sigma_{\mathrm{h} 2} \quad$ : contraintes horizontales ou radiales.

$\sigma_{0} \quad:$ contrainte orthoradiale

$\sigma_{v} \quad$ : contrainte verticale ou dans l'axe du forage.

\section{Valeurs numériques}

$\mathrm{K}_{\mathrm{n}}=2000 \mathrm{Mpa}$

$\mu=10^{-3} \mathrm{~Pa} . \mathrm{S}$

$\rho_{\mathrm{o}}=10^{3} \mathrm{~kg} / \mathrm{m}^{3}$

$\Phi=2 \%$

$v_{0}=0,22$

$\mathrm{K}_{\mathrm{b}}=49700 \mathrm{Mpa}$

$\mathrm{G}_{\mathrm{b}}=22800 \mathrm{Mpa}$

$L_{1}=280 \mathrm{~mm}$

$\mathrm{R}_{1}=15 \mathrm{~mm}$

$\mathrm{R}_{\mathrm{o}}=250 \mathrm{~mm}$

\section{Introduction}

Dans le cadre des études de sûreté liées au stockage des déchets radioactifs en formation géologique, l'Institut de Protection et de Sûreté Nucléaire (IPSN) a confié au Laboratoire de Mécanique de Lille (L.ML), qui est doté d'un appareillage triaxial vrai de grande capacité, l'étude de la variation de la perméabilité d'un granite soumis à une sollicitation de contraintes variables.

La sollicitation triaxiale exercée sur l'échantillon (Fig. 1), successivement croissante puis décroissante, est caractérisée par trois contraintes totales principales : $\sigma_{\mathrm{v}} \sigma_{\mathrm{h} 1}, \sigma_{\mathrm{h} 2}$. Le bloc testé est foré pour permettre l'injection d'eau; la contrainte $\sigma$, est orientée suivant l'axe du forage et représente la contrainte verticale.

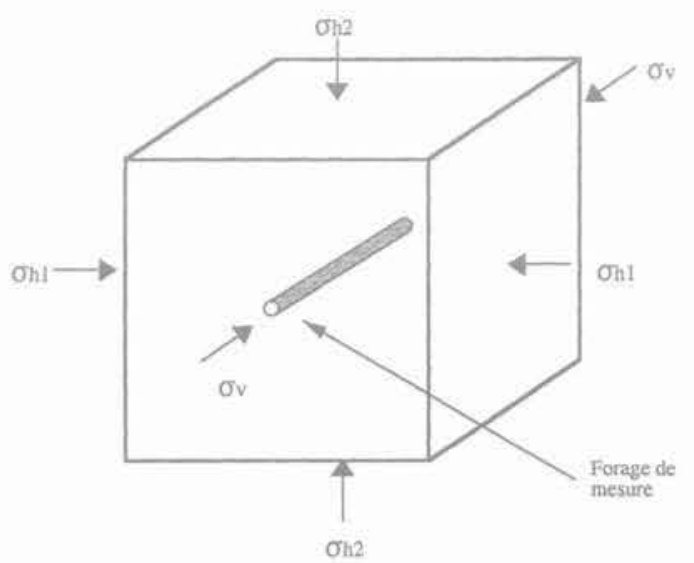

Fig.1 Schéma de la sollicitation exercée sur le bloc. Stresses applied on the block.

L'objectif de cette étude est de vérifier expérimentalement par des essais en laboratoire la loi empirique déduite d'essais in situ: $\mathrm{K}=\mathrm{K}_{0} \mathrm{e}^{- \text {tov }}$ [1], [2], [3],

$\mathrm{K}$ : perméabilité à la profondeur z;

$\mathrm{K}_{\mathrm{a}}$ : perméabilité à $\mathrm{z}=0$;

$\sigma_{\mathrm{v}}$ : contrainte effective verticale;

$\alpha$ : paramètre dépendant de la nature de la roche et de la fracturation.

Pour atteindre cet objectif divers types de mesures ont été mises au point. Deux types d'essais ont été pratiqués pour mesurer la perméabilité: en régime d'écoulement permanent et en régime transitoire par pulsetests. Le deuxième type d'essai ne permet pas une mesure directe de $\mathrm{K}$ et a nécessité un traitement numérique des résultats.

La loi empirique présentée plus haut suppose un état de contrainte horizontal homogène qui n'est pas vérifié près du forage d'injection. Nous proposons dans le cadre de cet article une modification de cette loi tenant compte de la variation importante de la contrainte orthoradiale près du forage. 


\section{Principe des mesures}

\section{1}

\section{Équation de diffusion. Principe de la mesure en régime permanent}

Nous supposons que le matériau rocheux est homogène, isotrope, parfaitement saturé, et que l'écoulement est régi par la loi de Darcy. La vitesse de diffusion V du fluide est alors donnée par:

$$
\mathrm{V}=-\frac{\mathrm{K}}{\mu} \operatorname{grad} \mathrm{P}
$$

avec: $\mu$ viscosité dynamique du fluide en Pa.s;

K perméabilité intrinsèque en $\mathrm{m}^{2}$,

$\mathrm{P}$ pression interstitielle en $\mathrm{Pa}$.

La notation employée ici est celle de la perméabilité intrinsèque que l'on peut relier à la conductivité hydraulique. Pour de l'eau à $20^{\circ} \mathrm{C}$, on a la correspondance $\mathrm{K}=1 \mathrm{~m}^{2}$ avec la conductivité hydraulique $\mathrm{k}=10^{7} \mathrm{~m} / \mathrm{s}$.

En régime d'écoulement permanent la conservation de masse s'écrit:

$$
\operatorname{div}(\rho \mathrm{V})=0
$$

Le dispositif expérimental décrit plus loin est conçu pour réaliser un écoulement de géométrie cylindrique (Fig. 2).

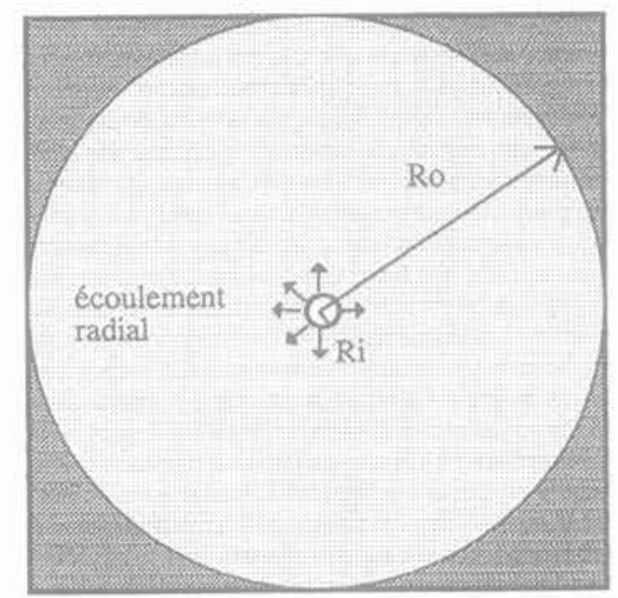

Fig. 2 Géométrie de l'écoulement. Cylindrical flow.

Dans le cadre de cette géométrie les variables seront des fonctions du seul rayon r. Dans l'hypothèse où $\mathrm{K}$ est uniforme et obéit à la loi empirique donnée précédemment, les équations (1) et (2) donnent la pression dans le domaine P:

$$
\mathrm{P}=\left(\mathrm{P}_{1}-\mathrm{P}_{\mathrm{o}}\right) \frac{\log \frac{\mathrm{r}}{\mathrm{R}_{0}}}{\log \frac{\mathrm{R}_{\mathrm{i}}}{\mathrm{R}_{\mathrm{o}}}}+\mathrm{P}_{\mathrm{o}}
$$

avec: $\mathrm{P}$ pression d'injection;

$\mathrm{P}_{0}$ pression au rayon $\mathrm{R}_{\mathrm{a}}$.
$R_{0}$ représente le rayon de drainage non perturbé en pression donc $\mathrm{P}$ y est nulle. L'équation (3) peut alors s'écrire différemment par:

$$
\mathrm{P}=\frac{\mu \mathrm{Q}}{2 \pi \mathrm{L}_{\mathrm{i}} \mathrm{K}} \log \frac{\mathrm{r}}{\mathrm{R}_{\mathrm{o}}}
$$

avec: Q débit d'injection;

$\mathrm{L}$ longueur du forage.

De l'équation (4) on obtient la perméabilité K:

$$
K=\frac{\mu Q}{2 \pi L / P_{i}} \log \frac{R_{j}}{R_{0}}
$$

La mesure de K repose sur la connaissance du débit d'injection Q ou du volume d'eau injecté pendant un temps donné en supposant que $Q$ est constant.

Pour mesurer précisément ce volume, l'eau injectée est pompée dans des capillaires calibrés. La méthodologie de l'essai peut donc être décomposée en six phases:

- saturation prolongée de l'échantillon (à $\left.\mathrm{P}_{1}=1,5 \mathrm{MPa}\right)$; - mise en place dans le triaxial vrai et application des contraintes $\sigma_{v} \sigma_{\mathrm{h}}$

- saturation sous contraintes:

- pompage dans un capillaire calibré pour mesurer le débit moyen d'injection pendant un temps t fixé;

- calcul de $K$ avec $Q=Q_{\text {mov }}$;

- passage à un autre état de contraintes.

La perméabilité obtenue sera ici un K apparent car son calcul repose sur l'hypothèse de son uniformité.

Dans le cas contraire le profil $\mathrm{P}(\mathrm{r})$ donné par l'équation (3) n'est plus correct et dépend de la fonction K(r) choisie. En nous appuyant sur la loi empirique précédente nous choisissons $\mathrm{K}$ de la forme :

$$
\mathrm{K}=\mathrm{K}_{\mathrm{o}} \mathrm{e}^{\mathrm{ft}(\mathrm{x})}
$$

$\mathrm{f}(\mathrm{r})$ sera précisée plus loin lors de l'étude des résultats obtenus. L'équation de conservation de la masse et la loi de Darcy aboutissent à l'équation différentielle suivante:

$$
\frac{d^{2} P}{d r^{2}}+\frac{d P}{d r}\left(\frac{1}{r}+\frac{d f(r)}{d r}\right)=0
$$

Nous intégrons cette équation par:

$$
P(r)=\lambda \int_{R j}^{r} \frac{1}{t} e^{-f(t)} d t+P_{j}
$$

$\lambda$ est une constante d'intégration telle que $\mathrm{P}(\mathrm{R})$ soit égale à $\mathrm{P}_{0}$. Nous pourrons l'évaluer numériquement quand la fonction $\mathrm{f}(\mathrm{r})$ sera définie et connaître ainsi $\mathrm{dp} / \mathrm{dr}$ au rayon $\mathrm{R}_{\mathrm{p}}$. La loi de Darcy (1) et la mesure du débit injecté $\mathrm{Q}$ permettent alors le calcul de K qui sera la perméabilité au bord du forage notée $K$. La perméabilité dans le domaine pourra finalement être déduite de l'équation (6).

\section{2.}

\section{Loi de comportement. Équation de diffusivité hydraulique}

Le granite testé est un matériau de faible porosité (environ $2 \%$ ). Nous supposons ici que son comportement mécanique est celui d'un matériau poro-élastique linéaire isotrope dont Biot [1] a établi la loi de comportement: 


$$
\begin{aligned}
\sigma_{i j} & =\sigma_{i j}^{o}+\left(K_{b}-\frac{2 G_{b}}{3}\right) \varepsilon_{k k} \delta_{i j} \\
& +2 G_{b} \varepsilon_{i j}-\beta\left(P-P_{0}\right) \delta_{i j}
\end{aligned}
$$

avec:

$\sigma^{\circ}$ état de contrainte initial;

$\mathrm{K}_{\mathrm{b}}$ module d'incompressibilité drainé;

$\mathrm{G}_{\mathrm{h}}$ module de cisaillement drainé;

$\beta$ coefficient de Biot.

Si la perméabilité est uniforme, l'équation de diffusivité [8] régissant l'écoulement s'écrit:

$$
\frac{1}{\mathrm{M}} \frac{\partial \mathrm{P}}{\partial \mathrm{t}}+\beta \frac{\partial \varepsilon_{\mathrm{kk}}}{\partial \mathrm{t}}=\frac{K}{\mu} \nabla^{2} \mathrm{p}
$$

avec: $M$ module de Biot;

$\nabla^{2}$ Laplacien de P.

Cette équation introduit ici le couplage poro-mécanique par le terme $\beta \frac{\partial \varepsilon_{k k}}{\partial t}$ dont nous verrons plus loin la faible influence. $\frac{\partial \varepsilon_{k k}}{\partial t}$ est due à l'évolution de la contrainte effective moyenne $\frac{\partial \sigma_{k k}^{i}}{\partial t}$ qui ne dépend que de la variation de pression P par $\sigma_{k k}^{\prime}=\sigma_{k k}-3 \beta P . \sigma_{k k}$ étant constant et $\beta$ faible [4], $\sigma_{k \mathrm{k}}^{\prime}$ va très peu évoluer.

\section{3}

\section{Principe du pulse-test}

Pour mesurer les très faibles perméabilités, une technique est souvent employée : le pulse-test [8]. Cet essai doit s'appliquer à un échantillon saturé et initialement soumis à un écoulement en régime permanent avec une pression $P_{i}^{\circ}$ dans le forage. Une injection rapide dans celui-ci permet une montée en pression jusqu'à la valeur $\mathrm{P}^{3}$. L'injection est ensuite stoppée, et il est possible de mesurer l'évolution de la pression en fonction du temps $\mathrm{P}(\mathrm{t})$. La figure 3 présente une telle évolution. Sans traitement numérique, la mesure $\mathrm{P}(\mathrm{t})$ ne permet pas le calcul direct de la perméabilité. Aux équations (9) et (10), valables en tout point de la roche, il faut ajouter celles qui traduisent la conservation de masse fluide dans le forage, et relier la quantité de fluide injectée à la variation de volume du forage.

Dans la résolution présentée ici nous n'avons pas tenu compte de la non-uniformité de K, cette étude peut donc être considérée comme une première approche nécessitant d'être affinée en introduisant la variation spatiale de K dans l'équation (10) et dans la résolution numérique de cette équation. Les résultats donnés par cette approche pourront néanmoins être comparés au $\mathrm{K}$ apparent mentionné précédemment.

Lors d'un écoulement radial, au rayon $\mathrm{R}_{1}$, la vitesse de diffusion $V_{1}$ s'écrit:

$$
V_{i}=-\frac{K}{\mu} d_{i} P
$$

avec:

$$
\mathrm{d}_{\mathrm{i}} \mathrm{P}=\frac{\partial \mathrm{P}}{\partial \mathrm{r}}(\mathrm{r}=\mathrm{Ri})
$$

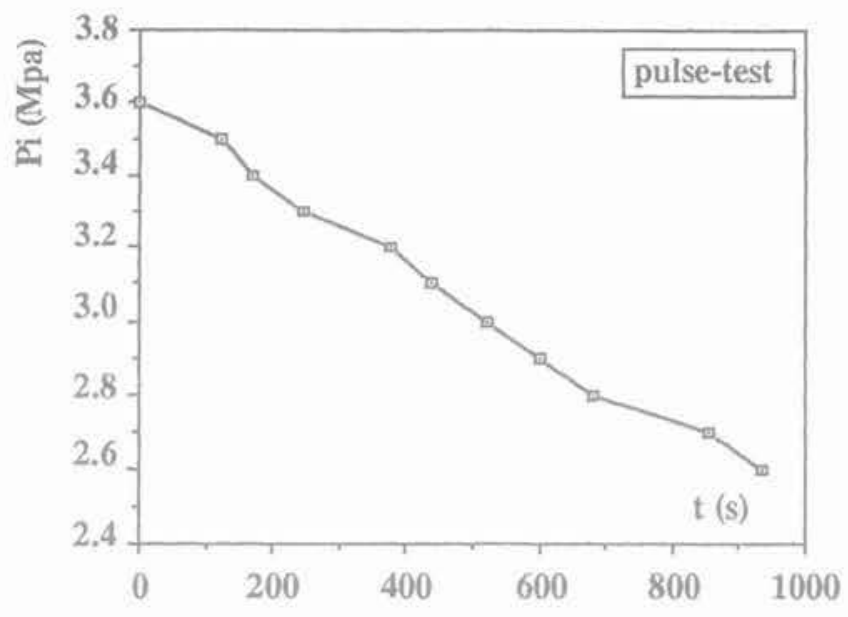

FG.3 Exemple de l'évolution de la pression dans le forage pour un pulse-test. Example of pressure variation in the borehole during a pulse test. vaut:

La masse de fluide injectée pendant le temps dt

$$
d m=-\rho_{i}(t) \frac{K}{\mu} \cdot d_{i} P \cdot S_{i} \cdot d t
$$

avec: $p(t)$ masse volumique du fluide à la pression $\mathrm{P}(\mathrm{t})$; $\mathrm{S}$ surface intérieure du forage.

Si $\mathrm{K}_{\mathrm{fl}}$ est le module d'incompressibilité volumique du fluide nous pouvons exprimer $p_{i}(t)$ par:

$$
p_{i}(t)=p_{0}\left(1+\frac{P_{1}(t)-P_{0}}{K_{f 1}}\right)
$$

$\rho_{0}$ masse volumique à la pression $\mathrm{P}$.

Nous notons $\Theta$ le volume de la chambre d'injection (volume du forage et des tuyauteries) et nous posons que la variation $\mathrm{d} \Theta$ liée à la variation de pression $\mathrm{dP}$ s'écrit:

$$
\frac{\mathrm{d} \Theta_{\mathrm{i}}}{\Theta_{\mathrm{i}}}=\frac{\mathrm{dP}}{\mathrm{K}_{\mathrm{y}}}
$$

$\mathrm{K}$ traduit ici la compressibilité du montage (tuyauterie et forage). Le volume de la tuyauterie, très faible devant celui du forage, est négligeable. K peut être alors calculé en exprimant le champ de déplacement $\mathrm{dU}(\mathrm{r})$ du domaine pour les conditions imposées. Pour ce type de chargement, dU(r) peut être exprimé [6] par :

$$
\frac{r v_{b}}{\lambda_{b}} d P_{i}\left[\frac{R_{i}^{2}}{R_{0}{ }^{2}-R_{i}^{2}} \cdot \frac{1}{r^{2}} \frac{R_{0}^{2} R_{i}^{2}}{R_{0}^{2}-R_{i}^{2}} \cdot \frac{1}{1-2 v_{b}}\right] \text { (15) }
$$

En négligeant ici $R_{1}^{2}$ devant $R_{0}^{2}$ la variation relative de volume calculée avec l'équation (15) s'écrit:

$$
\frac{d \Theta_{1}}{\Theta_{i}}=\frac{d P_{i}}{G_{b}}
$$

Pendant un temps dt la masse de fluide contenue dans le forage a varié de $d\left(\rho_{i} \Theta_{i}\right)$ soit:

$$
d m=\rho_{0} \Theta_{i} \frac{d P_{i}}{K_{f l}}+\rho_{o}\left(1+\frac{P_{i}-P_{0}}{K_{f l}}\right) \frac{d P_{i}}{G_{b}} \Theta_{l}
$$


En remarquant que $\left(\mathrm{P}_{1}-\mathrm{P}_{0}\right) / \mathrm{K}_{n}$ est très petit devant l'unité et en comparant les équations (17) et (12), il est possible de calculer la valeur de K par:

$$
\mathrm{K}=\mu \frac{\mathrm{L}_{\mathrm{i}}}{\mathrm{d}_{\mathrm{i}} \mathrm{P}}\left[\frac{1}{\mathrm{~K}_{\mathrm{fl}}}+\frac{1}{\mathrm{G}_{\mathrm{b}}}\right] \frac{\mathrm{dP}}{\mathrm{dt}}
$$

Nous observons que la perméabilité est fonction de la chute de pression $\mathrm{dP} / \mathrm{dt}$ et du gradient de pression $\mathrm{dP}$ au bord du forage. La dérivée $\mathrm{dP} / \mathrm{dt}$ est ici une donnée expérimentale indirecte puisque l'on mesure $\mathrm{P}(\mathrm{t})$.

Cette fonction $\mathrm{P}(\mathrm{t} t)$ peut être considérée comme une condition aux limites connue. L'inconnue sera le gradient de pression d. $\mathrm{P}$ qui ne peut être calculé qu'en résolvant l'équation (10) qui gouverne la diffusivité hydraulique. Le calcul de K passe par cette résolution dont la solution dépend de $K$. On peut donc envisager ici un calcul de la perméabilité de type itératif en deux phases distinctes :

1 - une phase de montée rapide en pression de $\mathrm{P}_{i}^{0}$ à $\mathrm{P}_{i}^{1}$; L'amplitude du pulse-test est la différence $\mathrm{P}^{1}-\mathrm{P}^{0}$; 2 - une phase de dissipation de la pression donnée par l'évolution $\mathrm{P}(\mathrm{t})$.

\section{Principe de calcul de la perméabilité}

Le principe de calcul de $\mathrm{K}$ repose ici sur la résolution simultanée des équations (9), (10) et (18) si l'on veut effectuer un calcul couplé poromécaniquement. Pour une perméabilité donnée $K_{\alpha}$ les équations (9) et (10) permettent le calcul de $\mathrm{P}(\mathrm{r}, \mathrm{t})$ en tout point du domaine. La résolution complète, par éléments finis, du problème thermoporoélastique a été présentée par F Skoczylas et al. [7] et nous en reprenons l'essentiel sans tenir compte des effets thermiques. Les conditions aux limites sont de type Dirichlet:

$$
\begin{aligned}
& P=P_{1}(t) \text { au rayon } R_{i} ; \\
& \sigma_{r}=-P(t) \text { au rayon } R_{j} ; \\
& \sigma_{r}=\text { contrainte radiale; } \\
& P=P_{0} \text { au rayon } R_{0} ; \\
& \sigma_{r}=\sigma_{h} \text { au rayon } R_{0} .
\end{aligned}
$$

Le calcul est incrémental du point de vue temporel et itératif pour la perméabilité. L'équation (18) est utilisée pour converger vers une valeur compatible de $\mathrm{K}$ pour le calcul de gradient d.P. Le calcul se déroule ainsi :

1 - phase de montée rapide en pression jusqu'à $\mathrm{P}^{1}$ avec $\mathrm{K}=\mathrm{K}$, Cette phase très rapide est indépendante du reste de la résolution, et la valeur de $\mathrm{K}$ initiale choisie pour cette étape n'influe pas sur la suite du calcul. Il est néanmoins possible après une première estimation de $\mathrm{K}$ réel de revenir sur cette phase, puis à chaque incrément de temps;

2 -résolution de (10) avec les conditions aux limites définies plus haut et calcul du gradient $\mathrm{d}_{\mathrm{l}} \mathrm{P}$;

3 - calcul de $K$ avec l'équation (18) et la valeur de dP. Ce calcul fournit $\mathrm{K}=\mathrm{K}_{1}$;

4 - retour sur la phase 2 avec $\left(K=K_{1}\right)$ et poursuite des étapes 2 et 3 jusqu'à convergence sur K;

5 - passage à l'incrément de temps suivant.
Si on se fixe un temps de résolution donné, la perméabilité finale sera prise comme la moyenne de l'ensemble des perméabilités obtenues à chaque incrément de temps.

L'expérience a montré que les résultats étaient généralement satisfaisants (bonne corrélation entre la mesure en régime permanent et par pulse-test) quand. on limitait le temps de résolution à un temps correspondant au début de l'essai (de l'ordre de 5 à $10 \%$ du temps total).

\section{3}

\section{Dispositif expérimental}

\section{1}

\section{Description de l'essai}

Le but d'un essai consiste à mesurer la variation de la perméabilité d'un bloc cubique de granite sous sollicitation triaxiale. Le bloc quil est un cube de $50 \mathrm{~cm}$ de côté est soumis à des chargements croissants puis décroissants. L'appareillage utilisé est le triaxial vrai du Laboratoire de Mécanique de Lille qui permet d'exercer des contraintes maximales, par axe, de $70 \mathrm{MPa}$ (sur ce type de bloc). L'eau est injectée dans un forage réalisé dans le bloc, de $30 \mathrm{~mm}$ de diamètre et de $280 \mathrm{~mm}$ de longueur entre obturateurs (Fig. 4). Deux blocs ont été testés: un bloc sain et un bloc traversé par une macrofissure dont le plan est perpendiculaire à l'axe du forage.

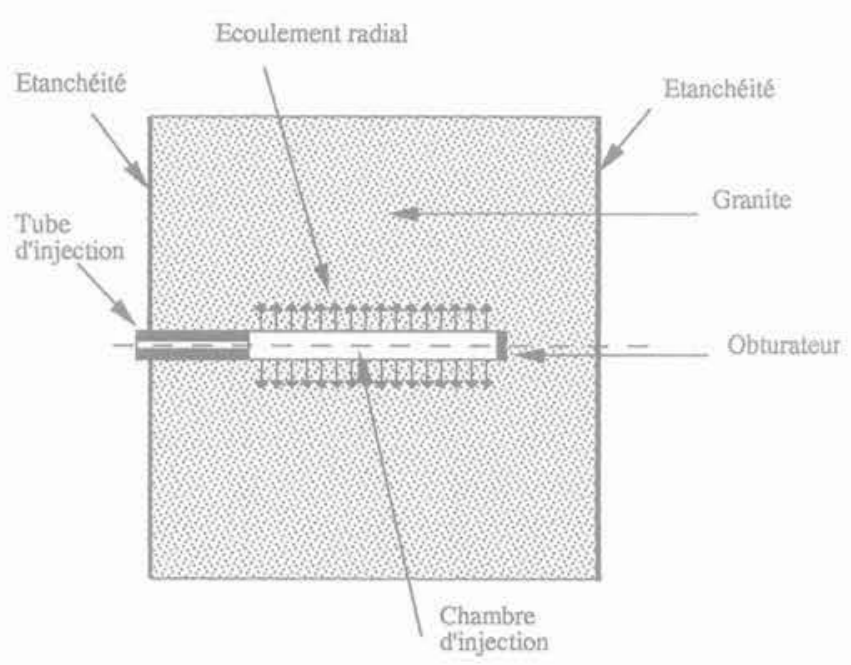

FIG.4 Préparation de l'échantillon. Sample preparation.

Le bloc est placé dans l'appareil et est soumis simultanément à trois contraintes $\sigma_{v}, \sigma_{h y}, \sigma_{h 2}$ et à une injection d'eau dans le forage. Les faces du bloc perpendiculaires à l'axe du forage sont étanchées, les autres faces sont librement drainées. Ce dispositif permet d'obtenir un écoulement le plus proche possible de l'écoulement radial. Une injection de colorant, suivie d'un découpage du bloc, a permis la vérification a posteriori de cette hypothèse. 


\section{Déroulement de l'essai}

La phase de mesures ne débute qu'après une longue saturation, 3 à 5 semaines, du bloc de granite. La contrainte $\sigma_{\mathrm{y}}$ est ensuite appliquée par paliers de 3 à $5 \mathrm{MPa}$. Les contraintes $\sigma_{h, 1}$ et $\sigma_{h,}$ sont égales à $\sigma_{h}$, et on peut définir le rapport $\mathrm{k}_{0}\left(\sigma_{h} / \sigma_{\mathrm{v}}\right)$ qui reste constant pendant un cycle de chargement-déchargement. Dès que le débit d'injection est stable, une mesure de perméabilité est effectuée pour chaque palier de chargement. Deux types de mesures sont réalisées : en régime permanent avec une pression d'injection constante $\mathrm{P}_{1}=$ 1,5 MPa ou une mesure par pulse-test. Lors d'un essai en régime permanent, le débit d'injection moyen est donné par une mesure précise du volume d'eau pompé dans des capillaires calibrés, Le tableau I résume l'ensemble des essais réalisés.

TABLEAU Récapitulatif des tests effectués.

\begin{tabular}{c|c|c|c} 
Bloc n & $\begin{array}{c}\sigma_{\text {mini }} \\
(\mathrm{MPa})\end{array}$ & $\begin{array}{c}\sigma_{\text {maxi }} \\
(\mathrm{MPa})\end{array}$ & $k_{0}$ \\
\hline $\begin{array}{c}1 \\
\text { fissuré }\end{array}$ & 5 & 33 & 0,7 \\
\hline 2 & 3 & 32 & 0,5 \\
sain & 3 & 30 & 0,7 \\
\hline 3 & 5 & 17,5 & 2 \\
\hline sain & 1,9 & 40 & 1 \\
\hline
\end{tabular}

\section{4}

\section{Résultats}

Les annexes 1 et 2 présentent l'ensemble des résultats obtenus sur trois blocs différents. Nous en reprenons ici l'essentiel.

\section{1}

\section{Chargement isotrope: $k_{0}=1$}

Nous présentons ici les résultats obtenus pour un bloc sain (sans fissure apparente) soumis au chargement isotrope $\sigma_{y}=\sigma_{h}$. Il n'est pas tenu compte de l'état non homogène de contraintes qui règne près du forage. Ce point fera l'objet d'une étude particulière, présentée plus loin, prenant en compte une variation de la perméabilité avec le rayon $\mathrm{r}$. La perméabilité $\mathrm{K}$ a donc été calculée en la supposant constante dans l'échantillon et peut être considérée comme une perméabilité apparente.

Les deux types d'essais ont été pratiqués: régime d'écoulement permanent et pulse-test, auxquels il faut ajouter quelques tests comparatifs en cellule de confinement. Dans le cas du pulse-test une étude paramétrique sur l'influence du coefficient de Biot intervenant dans l'équation (10), a été menée afin de vérifier pour un matériau aussi peu poreux le rôle négligeable du couplage poro-mécanique sur l'évolution de la pression $\mathrm{P}(\mathrm{r}, \mathrm{t})$ dans le domaine.

\section{Régime d'écoulement permanent}

Le bloc est chargé jusqu'à une contrainte uniforme $\sigma_{v}=\sigma_{b}=40 \mathrm{MPa}$, puis déchargé par paliers jusqu'à $15 \mathrm{MPa}$. La figure 5 montre l'évolution de la perméabilité en fonction du chargement. Un phénomène d'hystérésis au cours du déchargement apparait assez nettement, mais tend à disparaitre si on laisse un temps beaucoup plus long ( 7 à 10 jours) entre deux paliers de déchargement.

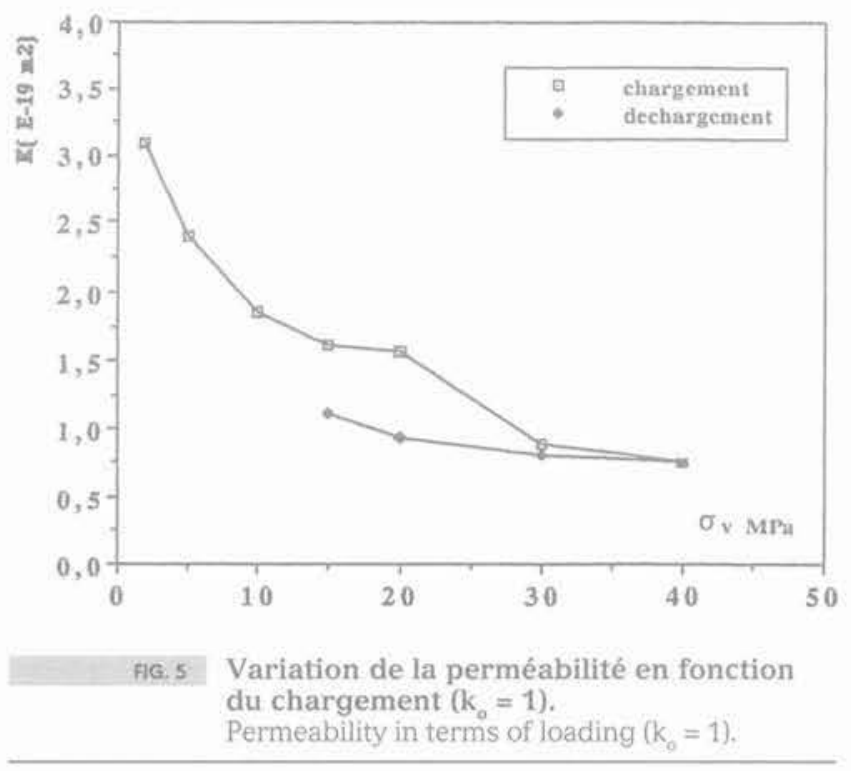

A titre de comparaison, le tableau II présente les résultats obtenus sur un échantillon cyclindrique. La perméabilité matricielle mesurée sur cet échantillon est plus faible que celle du bloc tout en étant du même ordre de grandeur (entre $10^{-19}$ et $10^{-20} \mathrm{~m}^{2}$ ). Ce résultat peut s'expliquer par un effet d'échelle favorable à l'échantillon cylindrique dont on peut plus facilement contrôler l'absence de microfissuration. La variation de $K$ en fonction de la pression de confinement $\mathrm{P}_{c}$ dans la cellule peut être exprimée avec une bonne précision par :

avec :

$$
\mathrm{K}=\mathrm{K}_{0} \mathrm{e}^{-\alpha i c}
$$

$$
\begin{gathered}
\mathrm{K}_{0}=5,4 \times 10^{-20} \mathrm{~m}^{2} \\
\alpha=0,067 \\
\mathrm{P}_{c} \text { en } \mathrm{MPa}
\end{gathered}
$$

TABLEAUIII Résultats obtenus en cellule de confinement.

\begin{tabular}{l|c|c|c|c|c}
\hline$P_{c}(\mathrm{Mpa})$ & 5 & 8 & 11 & 14 & 20 \\
\hline $\mathrm{K}\left(10^{-20} \mathrm{~m}^{2}\right)$ & 3,85 & 3,5 & 2,4 & 1,91 & 1,46 \\
\hline
\end{tabular}

Le coefficient $\alpha$ trouvé pour cet essai sera utilisé plus loin dans l'expression $\mathrm{K}=\mathrm{K}_{0} . \mathrm{f}(\mathrm{r})$ que nous avons à déterminer pour un dépouillement plus précis des essais. 


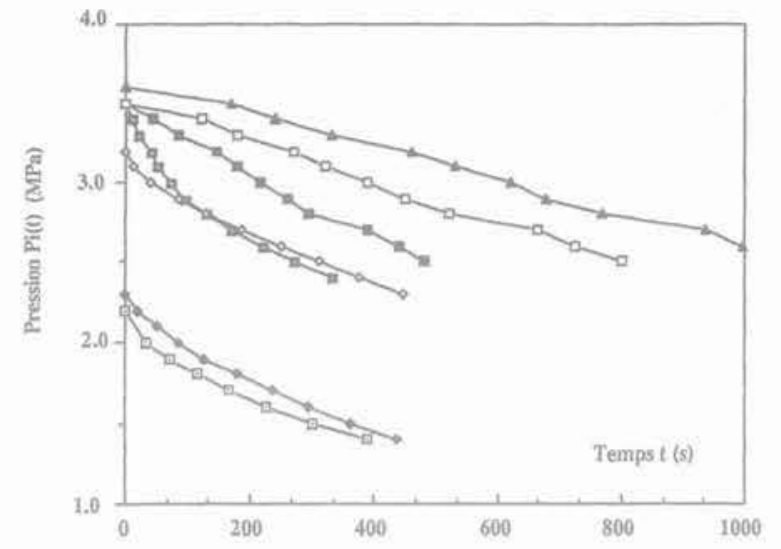

Fig.6 Chute de pression pour l'ensemble des pulse-tests.

Pressure decrease for all pulse tests.

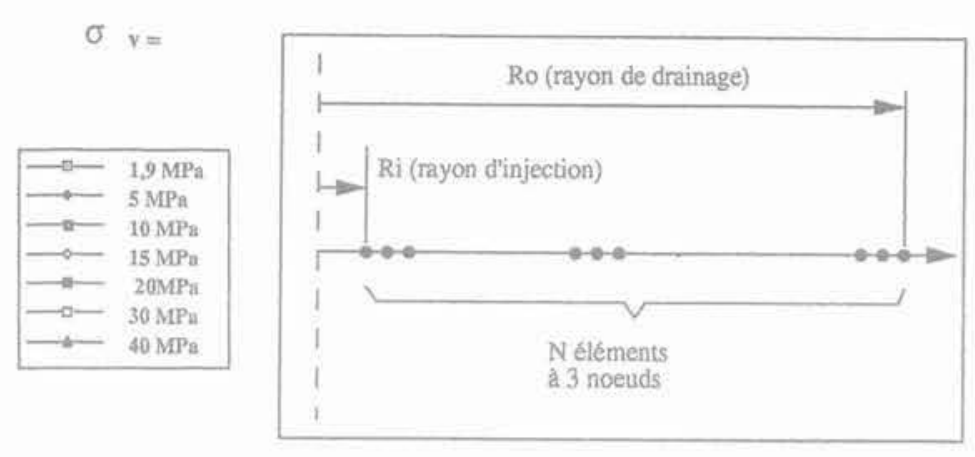

FG.7 Maillage du domaine. Domain mesh.

\section{$4.10^{2}$}

\section{Mesures par pulse-test}

Nous avons effectué une mesure de ce type pour chaque valeur $\sigma_{y}$. La figure 6 permet l'observation de la chute de pression $\mathrm{P}$ (t) pour l'ensemble des mesures. Selon les cas, la pression $\mathrm{P}^{0}$ est de 0,8 ou $1,5 \mathrm{MPa}$. Le tableau III présente les résultats obtenus selon les deux méthodes de mesure, et montre la bonne concordance entre ces deux types de tests (surtout ici pour les faibles perméabilités). Les ordres de grandeurs sont dans tous les cas très satisfaisants. Tous les résultats par pulsetest ont été obtenus en utilisant un maillage à vingt éléments unidimensionnels à trois nœuds (Fig. 7).

L'influence du couplage mécanique a été testée en faisant varier le coefficient de Biot du matériau, coefficient qui n'a pas été mesuré. Le module d'élasticité et le coefficient de Poisson de ce granite sont connus. Pour ce type de roche peu poreuse $(\Phi=2 \%), 0$. Coussy [4] a montré que le coefficient de Biot s'approche de la valeur de $\Phi$. Nous avons testé les trois valeurs : $\beta=\Phi / 2$; $\beta=5 \Phi / 2 ; \beta=5 \Phi$, en remarquant que la variation de $\beta$

TABLEAU III Comparaison des résultats obtenus par pulse-tests et en régime d'écoulement permanent-K en $10^{-19} \mathrm{~m}^{2}$

\begin{tabular}{c|c|c}
\hline$\sigma_{y} \mathrm{MPa}$ & $\begin{array}{c}\mathrm{K} \\
\text { r.permanent }\end{array}$ & $\mathrm{K}$ par pulse-test \\
\hline 1,9 & 3,1 & 5,8 \\
5 & 2,4 & 4,7 \\
10 & 1,85 & 5,3 \\
15 & 1,6 & 2,9 \\
20 & 1,55 & 1,48 \\
30 & 0,89 & 0,78 \\
40 & 0,74 & 0,52 \\
30 & 0,81 & 0,6 \\
20 & 0,94 & 0,87 \\
\hline
\end{tabular}

TABleau iv Influence du coefficient de Biot $(\beta)$ sur les valeurs calculées de $\mathrm{K}\left(\mathrm{en} 10^{-19} \mathrm{~m}^{2}\right)$.

\begin{tabular}{c|c|c|c|}
\hline$\sigma_{i}(\mathrm{MPa})$ & $\beta=0,01$ & $\beta=0,05$ & $\beta=0,1$ \\
\hline 15 & 2,92 & 2,90 & 2,88 \\
30 & 0,78 & 0,78 & 0,78 \\
\hline
\end{tabular}

influe sur l'équation de diffusivité hydraulique par une modification du module de Biot [4] qui s'écrit :

$$
\frac{1}{M}=\frac{(\beta-\phi)}{K_{\mathrm{s}}}+\frac{\phi}{K_{f l}}
$$

$K_{\text {c }}$ est le module d'incompressibilité de la matrice solide. Ce module n'est pas connu mais nous le prenons égal à $\mathrm{K}_{\mathrm{h}}$ étant donné la très faible porosité du granite. Le tableau IV montre quelques résultats comparatifs pour $\sigma_{v}=15$ et $30 \mathrm{MPa}$, et prouve ici que le couplage mécanique a des effets négligeables sur la diffusion de la pression interstitielle.

\section{2}

\section{Chargement non isotrope}

\subsection{4}

\section{Chargement sur bloc non fissuré}

Un second bloc a étê testé suivant les mêmes principes que ceux exposés précédemment et nous donnons ici les valeurs de la perméabilité apparente mesurée pour $\mathrm{k}_{0}=0,7$ et $\mathrm{k}_{\mathrm{o}}=0,5$.

Pour $\mathrm{k}_{\mathrm{o}}=0,5$, valeur choisie lors du premier test, la figure 8 montre la variation de la perméabilité en phase de chargement et de déchargement. Un effet d'hystérésis très net s'observe de nouveau, d'autant plus marqué que ce test représente la première mise en charge du bloc.

En phase de chargement, on peut donner une approximation raisonnable de la perméabilité par la fonction :

$$
K=2,36\left(10^{-19} \mathrm{~m}^{2}\right) \exp \left(-0,053 \sigma_{v}\right)
$$

avec : $\sigma_{v}$ en $\mathrm{MPa}$ 


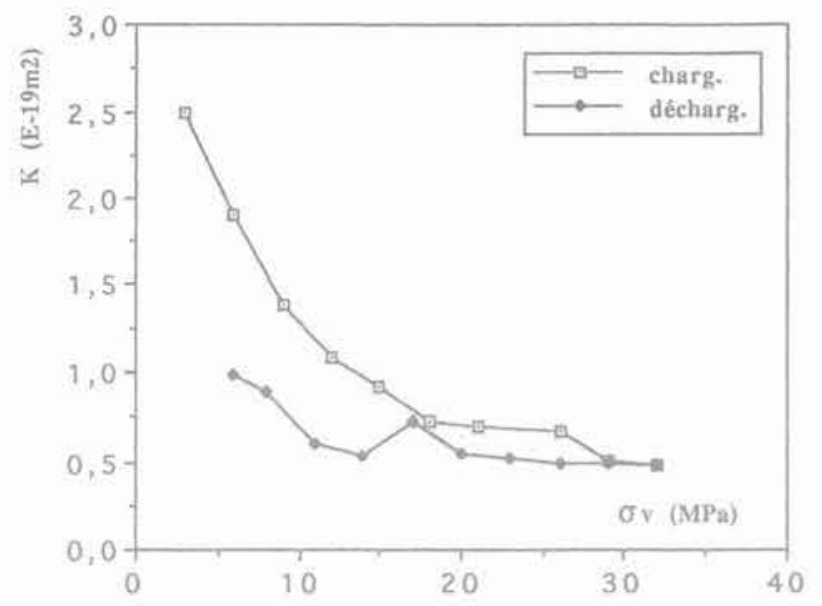

FiG. 8. Variation de la perméabilité en fonction de $\sigma_{\mathrm{y}}$ pour $\mathrm{k}_{0}=0,5$.

Permeability in terms of $\sigma_{\mathrm{r}}$ for $\mathrm{k}_{\mathrm{p}}=0.5$.

La variation de $\mathrm{K}$ pour un rapport $\mathrm{k}_{\mathrm{o}}=0,7$ est donnée par la figure 9. L'effet d'hystérésis sur trois points de décharge est ici absent. Cette observation semble montrer la nécessité d'une précharge du bloc avant tout test pour "stabiliser» le matériau. La première mise en charge peut certainement être associée à une fermeture irréversible de la microfissuration.

L'approximation de $\mathrm{K}$ est donnée ici par $\mathrm{K}=$ $1,34\left(10^{-19} \mathrm{~m}^{2}\right) \exp (-0,051 \sigma)$ et montre que tout en étant décalées les variations de $\mathrm{K}$ avec $\sigma_{\mathrm{u}}$ pour $\mathrm{k}=0,7$ et $\mathrm{k}_{\mathrm{a}}=0,5$ sont parallèles puisque de pentes très proches $(-0,053$ et $-0,051)$, avec une perméabilité plus faible pour $k_{0}=0,7$. Ces valeurs sont des mesures apparentes de la perméabilité mais il est évident que la contrainte orthoradiale $\sigma \theta$ au bord du forage joue un rôle jusqu'à présent négligé dans les dépouillements. Un calcul élastique montre que $\sigma \theta$ peut être, en négligeant $\mathrm{P}$ devant $\sigma_{h}$, écrite sous la forme:

$$
\sigma_{0} \approx \sigma_{h}\left(1+\left(\frac{R_{1}}{r}\right)^{2}\right)
$$

Une compression orthoradiale égale à $2 \mathrm{k}, \sigma$ apparaît au bord du forage et aura pour effet de diminuer la perméabilité (tant que le matériau n'est pas endommagé). Il faut, pour évaluer la correction à apporter aux valeurs apparentes de $K$, estimer la valeur de la fonction $\mathrm{f}(\mathrm{r})$ de l'équation 6 et l'utiliser dans l'équation 8.

\section{8.?}

\section{Estimation empirique de $f(r)$}

Les essais effectués ne l'ont pas été dans le but d'estimer précisément $\mathrm{f}(\mathrm{r})$, ce point fera l'objet d'essais futurs sur d'autres blocs avec des variations indépendantes de l'état de contraintes. Nous pouvons cependant proposer une allure de la variation de $\mathrm{K}$ en fonction des contraintes en nous basant sur l'expérience acquise en cellule où $K$ pouvait s'écrire $K=K_{0} e^{-a p c}$.

Si l'on considère un écoulement perpendiculaire à un plan 1.2 (Fig. 10) soumis à l'état de contraintes principales $\left(\sigma_{1}, \sigma_{2}\right)$ on peut supposer en première approximation que $\sigma_{3}$ (ici $\sigma$ ) joue un rôle négligeable devant $\sigma$. et $\sigma_{2}$ et tenter d'écrire $K$ sous la forme $K\left(\sigma_{1}, \sigma_{2}\right)$. Nous choisissons ici la fonction :

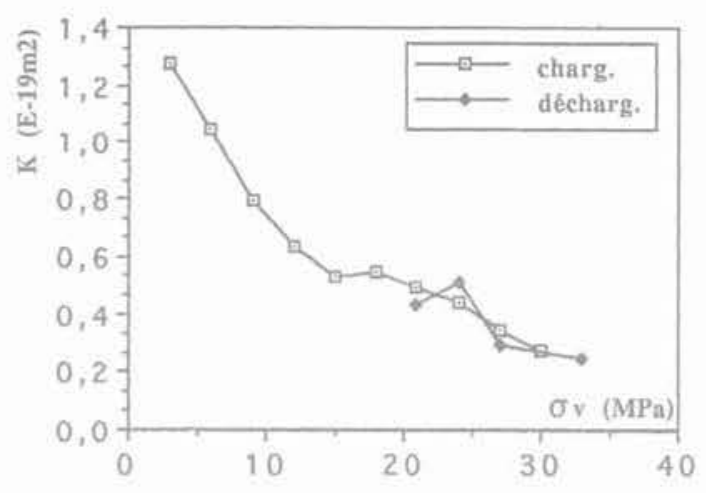

Fic. Variation de la perméabilité en fonction de $\sigma$ pour $\mathrm{k}_{0}=0,7$.

Permeability in terms of $\sigma_{\mathrm{s}}$ for $\mathrm{k}_{\mathrm{o}}=0,7$.

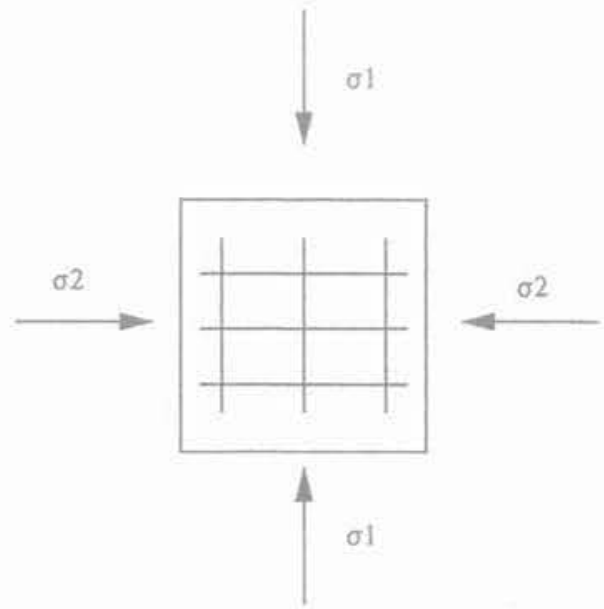

FG. 10 Plan de roche perpendiculaire à l'écoulement. Rock face perpendicular to the flow.

$$
K=K_{0} e^{-2 \alpha \frac{\sigma_{1} \sigma_{2}}{\sigma_{1}+\sigma_{2}}}
$$

Le choix de ce type de fonction peut être justifié par quelques observations :

- si $\sigma_{1}=\sigma_{2}=P_{c}$ on retrouve l'expression de $\mathrm{K}$ donnée en cellule triaxiale;

- si $\sigma_{1}=0$ ou $\sigma_{2}=0$ alors $K=K$. Nous tenons à privilégier ici l'influence des fermetures de microfissures. En effet, si $\sigma=0$ les fissures orthogonales à cette direction restent ouvertes, et nous supposons que l'écoulement s'y produira préférentiellement.

K s'écrit avec ces hypothèses :

$$
K=K_{0} \exp \left(-2 \alpha \cdot \sigma_{v} \frac{k_{0}\left(r^{2}+R_{1}^{2}\right)}{\left(\left(1+k_{0}\right) r^{2}+k_{0} R_{i}^{2}\right)}\right)
$$

Nous retiendrons pour $\alpha$ la valeur trouvée en cellule soit $\alpha=+0,067$. Les équations (6) et (1) permettent alors de donner une valeur modifiée de la perméabilité au bord du forage. 


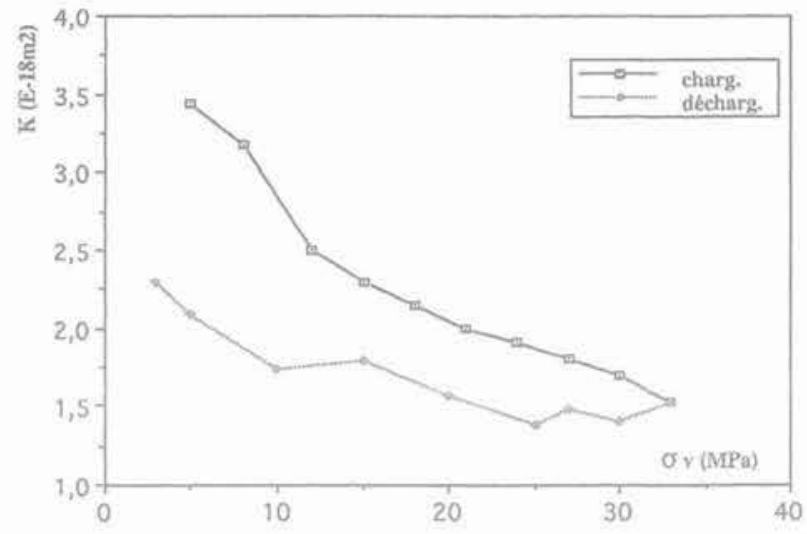

FiG. 11 Variation de la perméabilité en fonction de $\sigma$ pour le bloc fissuré.

Permeability in terms of $\sigma$ for the cracked block.
TABLEAUV Effets de la prise en compte

de la con-trainte orthoradiale sur la mesure de $\mathrm{K}$.

\begin{tabular}{|c|c|c|c|c|c|c|c|}
\hline & $\sigma_{y}(\mathrm{MPa})$ & 5 & 10 & 15 & 20 & 25 & 30 \\
\hline \multirow{3}{*}{$k_{0}=0,5$} & $\mathrm{~K}_{\mathrm{vp}}\left(10^{\left.-1 / \mathrm{m}^{2}\right)}\right.$ & 2,5 & 1,25 & 0,92 & 0,71 & 0,67 & 0,49 \\
\hline & $\mathrm{K}_{\text {mod }}\left(10^{-15} \mathrm{~m}^{2}\right)$ & 28 & 1,06 & 0,74 & 0,53 & 0,47 & 0,32 \\
\hline & $\mathrm{K}_{y}\left(10^{-11 m^{2}} \mathrm{~m}^{2}\right)$ & 3,13 & 2,03 & 1,94 & 1,94 & 2,35 & 2,28 \\
\hline \multirow{3}{*}{$k_{0}=0,7$} & $K_{e p}\left(10-m^{2}\right)$ & 1,1 & 0,7 & 0,51 & 0,49 & 0,43 & 0,27 \\
\hline & $\mathrm{K}_{\bmod }\left(10^{-1} \mathrm{~m}^{2}\right)$ & 1 & 0,59 & 0,40 & 0,36 & 0,3 & 0,18 \\
\hline & $\mathrm{K}_{v}\left(10^{-1} \mathrm{~m}^{2}\right)$ & 1,46 & 1,26 & 1,25 & 1,62 & 1,97 & 1,71 \\
\hline
\end{tabular}

TABlEAUA1 Résultats des mesures en phase de chargement pour les trois blocs testés

\begin{tabular}{|c|c|c|c|c|c|c|}
\hline$\sigma_{i}$ & $\begin{array}{c}1 \\
0,7 \\
\mathrm{~K} \\
\mathrm{E}^{-1} \mathrm{~m}^{2}\end{array}$ & $\begin{array}{c}2 \\
0,7 \\
K \\
E^{-1} m^{2}\end{array}$ & $\begin{array}{c}2 \\
0,5 \\
K \\
\mathrm{E}^{-19} \mathrm{~m}^{2}\end{array}$ & $\begin{array}{c}2 \\
2 \\
K \\
E-m^{2}\end{array}$ & $\begin{array}{c}3 \\
1 \\
K \\
E^{-12} m^{2}\end{array}$ & $\begin{array}{c}3 \\
0,7 \\
K \\
E-4 m^{2}\end{array}$ \\
\hline 2 & & & & & 3,1 & \\
\hline 3 & & 1,28 & 2,5 & & & \\
\hline 5 & 34,4 & & & 0,67 & 2,4 & \\
\hline 6 & & 1,05 & 1,9 & & & \\
\hline 7,5 & & & & 0,79 & & \\
\hline 8 & 31,7 & & & & & \\
\hline 9 & & 0,79 & 1,39 & & & \\
\hline 10 & & & & 0,36 & 1,85 & 1,43 \\
\hline 12 & 25 & 0,63 & 1,08 & & & \\
\hline 12,5 & & & & 0,28 & & \\
\hline 15 & 23 & 0,53 & 0,92 & 0,29 & 1,6 & \\
\hline 17,5 & & & & 0,32 & & \\
\hline 18 & 21,5 & 0,54 & 0,72 & & & \\
\hline 20 & & & & & 1,55 & 1,04 \\
\hline 21 & 20 & 0,49 & 0.7 & & & \\
\hline 24 & 19,1 & 0,44 & & & & \\
\hline 26 & & & 0,67 & & & \\
\hline 27 & 18 & 0,34 & & & & \\
\hline 29 & & & 0,51 & & & \\
\hline 30 & 17 & 0,27 & & & 0,89 & \\
\hline 32 & & & 0,48 & & & \\
\hline 33 & 15,2 & & & & & \\
\hline 40 & & & & & 0,74 & 0,73 \\
\hline
\end{tabular}

TABLEAUA2 Résultats des mesures en phase de déchargement pour les trois blocs testés/

\begin{tabular}{|c|c|c|c|c|c|c|}
\hline$\sigma_{v}$ & $\begin{array}{c}1 \\
0,7 \\
\mathrm{~K} \\
\mathrm{E}^{-19} \mathrm{~m}^{2}\end{array}$ & $\begin{array}{c}2 \\
0,7 \\
\mathrm{~K} \\
\mathrm{E}^{-1} \mathrm{~m}^{2}\end{array}$ & $\begin{array}{c}2 \\
0,5 \\
K \\
\mathrm{E}^{-1 \mathrm{l}^{2}} \mathrm{~m}^{2}\end{array}$ & $\begin{array}{c}2 \\
2 \\
\mathrm{~K} \\
\mathrm{E}^{-1 / 0} \mathrm{~m}^{2}\end{array}$ & $\begin{array}{c}3 \\
1 \\
K \\
E^{-15} m^{2}\end{array}$ & $\begin{array}{c}3 \\
0,7 \\
K \\
E^{-4} m^{2}\end{array}$ \\
\hline 3 & 23 & & & & & \\
\hline 5 & 20,9 & & & 0,63 & & \\
\hline 6 & & & 0,99 & & & \\
\hline 7,5 & & & & 0,42 & & \\
\hline 8 & & & 0,89 & & & \\
\hline 10 & 17,4 & & & 0,25 & & 1,16 \\
\hline 11 & & & 0,6 & & & \\
\hline 12,5 & & & & 0,41 & & \\
\hline 14 & & & 0,54 & & & \\
\hline 17 & & & 0,72 & & & \\
\hline 17,5 & & & & 0,332 & & \\
\hline 20 & 15.7 & & 0,54 & & 0,94 & 1,02 \\
\hline 21 & & 0,43 & & & & \\
\hline 23 & & & 0,52 & & & \\
\hline 24 & & 0,51 & & & & \\
\hline 25 & 13,9 & & & & & \\
\hline 26 & & & 0.5 & & & \\
\hline 27 & 14,8 & 0,29 & & & & \\
\hline 29 & & & 0,49 & & & \\
\hline 30 & 14,1 & & & & 0,81 & \\
\hline 32 & & & 0,48 & & & \\
\hline 33 & & 0,25 & & & & \\
\hline 40 & & & & & 0,74 & 0,73 \\
\hline
\end{tabular}




\section{3}

\section{Comparaison $\mathrm{K}$ apparente - $\mathrm{K}$ modifiée}

L'utilisation des équations (22) et (8) permet le calcul du gradient de $\mathrm{P}(\mathrm{r})$ au bord du forage pour chaque état de contrainte et de déduire les valeurs de la perméabilité modifiée. De ces résultats on peut également extraire la perméabilité matricielle pour le matériau vierge (sans contrainte) $\mathrm{K}_{0}$. Le tableau V donne l'ensemble de ces résultats qui montrent que la prise en compte de l'effet $\sigma_{\theta}(\mathrm{r})$ sur K (par l'équation (22)) aboutit à une réduction notable de la perméabilité au bord du forage. Cet effet était attendu, mais a surtout une valeur qualitative, puisqu'il repose sur la forme de l'équation 22 qu'il faudrait pouvoir préciser par d'autres essais.

Les résultats sur $\mathrm{K}_{0}$ sont intéressants puisque relativement homogènes pour chaque valeur de poussée $k_{0}$. On peut, toutefois, remarquer qu'il aurait fallu trouver la même valeur pour $\mathrm{K}$ dans les deux cas. Les différences obtenues peuvent s'expliquer par l'effet d'hystérésis et le choix de l'équation 22. La première valeur de $\mathrm{K}_{\mathrm{o}}$ pour $\mathrm{k}_{\mathrm{o}}=0,5$ peut être écartée, car elle correspond à la mise en charge initiale du bloc donc à une fermeture partielle de la microfissuration.

\section{$4,2.4$ \\ Bloc fissuré}

Cet échantillon présentait une macrofissure dans un plan perpendiculaire au forage. Les résultats obtenus qu'illustre la figure 11 montrent clairement l'importance de la fissuration sur la perméabilité qui est beaucoup plus élevée dans ce cas avec un rapport $\mathrm{K}$ (bloc fissuré)/K (bloc sain) de l'ordre de 30 environ, sans qu'une pression de fermeture complète ne soit atteinte. suration, en partie refermée, induit une perméabilité bien plus élevée que celle de la matrice du matériau.

Pour le bloc sain, l'approximation de la perméabilité $\mathrm{K}$ par une loi exponentielle en fonction de la seule contrainte $\sigma_{\mathrm{u}}$ montre ses limites puisque l'influence de la contrainte horizontale $\sigma_{h}$ (par l'intermédiaire de $\sigma_{\theta}$ au bord du forage) est très nette. Nous avons donc modifié en première approche; cette loi exponentielle par une loi empirique prenant en compte $\sigma_{y}$ et $\sigma_{\theta}$ et obtenu une valeur de la perméabilité plus faible que celle mesurée en la supposant uniforme dans le domaine. Néanmoins, il faut souligner le côté «artisanal» de cette loi qui doit être complétée par d'autres essais et intégrée dans le dépouillement des pulse-tests. Les essais sur bloc non fissurés montrent également des perméabilités très proches de celles mesurées en cellule de confinement.

Pour obtenir la perméabilité de ce granite d'autres types d'essais en écoulement transitoire ont été menés: les pulse-tests, Le dépouillement de ces tests a nécessité la mise au point d'un logiciel de calcul par éléments finis qui a pu être validé par comparaison avec les mesures fournies par la technique plus classique en régime d'écoulement permanent. Une étude paramétrique succincte a également montré que, pour ce matériau de très faible porosité, le couplage mécanique, déduit de la théorie de Biot, avait des effets négligeables sur la valeur de la perméabilité obtenue numériquement. Ces essais doivent être complétés par des mesures plus fines sur des cubes de granite pour déduire par des chemins de sollicitations indépendantes, des relations liant la variation de $\mathrm{K}$ avec le champ de contrainte et indirectement avec l'endommagement éventuel du matériau dont la perméabilité est un indicateur précoce.

En vue d'interpréter les résultats d'essais in situ, des méthodes nouvelles d'exploitation des pulse-tests au gaz ou à l'eau seront mises au point.

\section{5}

\section{Conclusion}

Les essais menés sur ces différents blocs ont permis de montrer l'influence de la fissuration sur la perméabilité, même à contrainte verticale très élevée. Cette fis-

\section{$\overline{\text { Bibliographie }}$}

[1] Biot M. - The theory of finite deformation of porous solids, Incliana Univ. Math

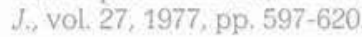

[2] Brace W.F. - A note on permeability changes in geological material due to stress, Pageoph., vol, 116, Birkhaüser Verlag, Basel, 1978, pp. 627-633.

[3] Carlsson A and Olsson T. - Variations of hydraulic conductivity in some swedish rock types, Rockstore 77 Stockholm Storage in excavated rock caverns, vol. 2, 1977, pp. 301-307.
[4] Coussy O. - Mécanique des milieux poreux, Paris, Éditions Technip., 1990.

[5] Louis Cl. - Rock Hydraulics, Rapport BRGM 74 SGN 035 AME, janvier 1974.

[6] Skoczylas F. - Contribution à l'étude de la distribution des contraintes autour d'un puits pétrolier. Application au calcul de la pression d'initiation de fracture hydraulique, Thèse de Doctorat, USTL Flandres-Artois, 1988.
[7] Skoczylas F., Shahrour I., Henry J.-P. Étude numérique du couplage thermohydromécanique dans les roches. Influence des termes de couplage non linéaires pour un matériau isotrope linéaire, Revue de IIFP, vol. 47, $\mathrm{n}^{\circ} 1$, janvier-février 1992, pp. 45-55.

[8] Walder J., Nur A. - Permeability measurement by the pulse-decay method: effects of poroelastic phenomena and non linear pore pressure diffusion, Int. J. Rock Mech. Min. Sci. \& Geomech. Abstr., vol. 23, 1986, pp. 225-232. 